\title{
Integrated health research centres for Australia
}

\section{Embedding research into health care organisations improves outcomes, but will require unique, flexible models for success}

$\mathrm{t}$ will come as little surprise to those who work in the health care sector that the Australian health system is highly fragmented. At the coalface, we have a wide variety of service providers, including public hospitals, primary care providers, aged care providers, private sector operators and many non-profit organisations and charities. Their work is complementary to, and interdependent with, the activities of teaching and research institutions such as universities and independent medical research institutes that help provide innovation and evidence-based models of illness prevention and health care.

Despite the interdependent nature of their mission, health services, universities and medical research institutes are funded separately, overseen by diverse governance structures, and subject to different measures of success - not all of which have better community health as an outcome. On closer inspection, what we actually have is three separate systems, and not three parts of one system.

With an ageing population and rapidly rising incidence of chronic disease, this model looks increasingly unsustainable and, more importantly, unaffordable.

A possible solution lies in the global trend to aggregate resources around centres of excellence with shared responsibility for tackling specific health conditions. One of the guiding principles of these arrangements is that research is embedded at every level, from teaching to delivery, with a view to establishing continuous improvements and, by extension, better health outcomes.

\section{Strategic review of health and medical research: better health through research}

The most recent review of health and medical research, chaired by Simon McKeon, provides an ambitious strategy for better health through a measured and consistent government approach that ensures a sustainable health and medical research sector. ${ }^{1}$ Among the recommendations of the report that are intended to help embed health research and development over time are: quarantining a proportion of health spending for health-related research and development; providing time for clinicians to pursue research; improving processes for clinical trials; and promoting health service research.

There are also recommendations that, if implemented, would go a long way towards improving the efficiency of health research spending. These are worthy ambitions and deserve bipartisan support. McKeon also
Garry L R Jennings
MD, FRACP,
Director

Michael K Walsh MB BS, BHA, MPA, Chief Executive, and Member, ${ }^{2}$ Member Council ${ }^{3}$

1 Baker IDI Heart and Diabetes Institute, Melbourne, VIC.

2 Cabrini Health, Melbourne, VIC.

3 Monash Partners Academic Health Science Centre, Melbourne, VIC.

garry.jennings@ bakeridi.edu.au

doi: 10.5694/mjal3.10141 recommends establishing and funding a limited number of integrated health research centres in Australia. The strategic review chaired by McKeon is the second national review to recommend embedding research in the health system as a way of improving health outcomes, ${ }^{2}$ and this recommendation is supported by international research showing that health organisations that undertake research produce better outcomes. ${ }^{3}$

\section{Is it time for Australian academic, advanced or integrated health research centres?}

For more than a century, United States centres that combine the activities of hospitals, universities and medical research institutes have been very successful in producing high-quality research and enhanced patient outcomes. Examples include Johns Hopkins Medicine, Partners HealthCare International (founded by Massachusetts General Hospital and Brigham and Women's Hospital, the two largest teaching hospitals of Harvard Medical School), Mayo Clinic and Stanford University School of Medicine. Academic health science centres (AHSCs) are analogous to integrated health research centres in McKeon terminology or advanced health science centres as described by the National Health and Medical Research Council, ${ }^{4}$ and emerged formally in the United Kingdom 5 years ago. In Australia, there are already a number of informal precinct-based AHSCs, but they lack the size and extent of private investment seen in other countries.

In moving toward a more effective and formal model of integration, we face a number of challenges. Funding and governance are perhaps the most complex obstacles to integration, and cannot be overcome with new logos and by simply superimposing an additional bureaucratic structure to act as a binding agent. We should also guard against the "merger and acquisition" approach that drove so much corporate activity in the 1980s and 90s — the assumption that a standardised approach would prevail in all markets and that cost efficiencies would naturally flow has since been shown to be flawed. Another trap is an exaggerated perception of synergy or even codependency of partners based on the convenience and efficiency of geographical collocation and historical ties.

There are still many issues to resolve before we plunge headlong into an overhaul of the status quo. In Australia, the private sector is responsible for more than half of all health care delivery. Would integrated health centres preclude large private hospitals because the funding models are too difficult to reconcile with government and non-profit arrangements? How might those with a vested interest, such as government and insurers, as well as consumers, be persuaded to pay for the outputs of an integrated health science centre, and what is the product 
that they are purchasing? What is the objective improved health of a local community or system-wide improvement through research and innovation? How much autonomy will individual members and, perhaps more importantly, their governing bodies, be willing to relinquish to the collective for priority-setting and resource allocation?

What is clear is that there is no single governance model that has been uniformly successful. ${ }^{1,4}$ Moreover, success (by any measure) is not guaranteed and there have been several failures internationally that we would do well to learn from.

To be effective, we will need to tailor the integrated health-science centre concept to the unique circumstances of the Australian setting. Integrated health research centres must be superimposed on, and take account of, the array of existing institutions in Australia involved in health care, health research and education, and their governing bodies. ${ }^{5}$ In Australia, we need to provide health care to a relatively small but widely dispersed population. We do this across, and within, multiple jurisdictions. The models that have served the US health and medical sector cannot simply be reproduced here, as these have largely been developed around elite private hospitals and universities. We are also different to the UK, where a single National Health Service allows integration of the various arms of the health system under a single master, albeit with multiple layers in between. Our strong privacy laws inhibit the flow of information needed for health service research.

\section{Beyond the capital city campus}

The establishment of integrated health science centres in Australia will need to embrace the move away from hospital-based treatment to prevention, community care and primary care. This flies in the face of existing paradigms of health integration, focused around large urban hospitals. However, it is critical that we identify a model that extends its benefits beyond elite centres in capital cities to community health services, where demand is often at its highest. While it is logistically more complicated, this has more synergy with the secular trend towards "big science" and large cohorts facilitated by the genomic revolution, where the number of people participating in a trial is now very much larger than in the past. Highly prevalent conditions like heart disease, cancer and diabetes need national and international strategies, and consortia rather than suburban marriages of convenience.

\section{Structures and governance}

Whatever governance structures we develop, they should be informed by - rather than dictate - accountability and performance metrics. Ideally, theme leaders within the partnerships would take responsibility for agreed and measurable deliverables, and be held accountable for performance. That performance should be aligned to funding incentives which service providers, educators and researchers can all respond to and be based, in part, on the health of the community they serve.

In the UK, the William Harvey Research Institute has wide responsibility not only for cardiovascular research, but also for clinical trials within the huge University College London Partners AHSC. At the Manchester AHSC, the Christie Hospital has the system-wide remit for cancer research. Allocation of responsibility for themes gives the group the advantage of having a champion working on each, and the individual institution the advantage of a system-wide remit. Everyone wins.

The review of medical research in Australia is an opportune time to think carefully about the role, structure, governance and the potential of integrated health science centres. Let's at least get the language right and talk about systems, networks and integration, not just precincts or centres. Evaluation of these initiatives with appropriate metrics and good baseline data will be essential to ensure that future investment is worthwhile, reduces transaction costs between institutions and ensures that efficient and timely research output benefits our community.

Acknowledgements: We acknowledge the assistance of Tracey Ellis, David Lloyd, Bronwyn Kingwell, Jenny Williams and Christina Hickie in the preparation of this article. Garry Jennings is supported by the National Health and Medical Research Council of Australia (grants 1000900 and 1036352) and the Operational Infrastructure Support Program of the State Government of Victoria.

Competing interests: Garry Jennings and Michael Walsh are heads of institutes that have joined Monash Partners Academic Health Science Centre.

Provenance: Not commissioned; externally peer reviewed.

1 Australian Government Department of Health and Ageing. Strategic review of health and medical research: better health through research. Canberra: Commonwealth of Australia, 2013. http://www.mckeonreview.org.au/ downloads/Strategic_Review_of_Health_and_Medical_Research_Feb_2013Final_Report.pdf (accessed Mar 2013).

2 National Health and Hospitals Reform Commission. A heathier future for all Australians - final report June 2009. Canberra: NHHRC, 2009. (NHHRC No. P35499.) http://www.yourhealth.gov.au/internet/yourhealth/publishing.nsf/ Content/nhhrc-report-toc\#.UVEkABxyCVI (accesssed Mar 2013)

3 Dzau VJ, Ackerly DC, Sutton-Wallace P, et al. The role of academic health science systems in the transformation of medicine. Lancet 2010; 375: 949-953.

4 National Health and Medical Research Council. Developing advanced health research centres in Australia. Discussion paper. Canberra: NHMRC, 2010. http://www.nhmrc.gov.au/research/advanced-health-research-centres (accessed Mar 2013).

5 Fisk NM, Wesselingh SL, Beilby JJ, et al. Academic health science centres in Australia: let's get competitive [editorial]. Med J Aust 2011; 194: 59-60.

\section{When too much MJA and MJA InSight is never enough.}

Follow us on Twitter (@theMJA) and like us on Facebook (https://www.facebook.com/theMJA) 